\title{
Resource allocation for ultra-reliable low latency communications in sparse code multiple access networks
}

\author{
Qinwei $\mathrm{He}^{1}$, Yulin $\mathrm{Hu}^{2 *}$ and Anke Schmeink ${ }^{1}$
}

\begin{abstract}
In this paper, we propose an optimal resource allocation policy for sparse code multiple access (SCMA) networks supporting ultra-reliable low-latency communications (URLLC). The network is assumed to operate with finite blocklength (FBL) codes, which is opposed to the classical information-theoretic works with infinite blocklength (IBL) codes. In particular, we aim at maximizing the average transmission rate in the FBL regime while guaranteeing the transmission reliability. A joint design is proposed, which combines the power allocation with the codebook assignment. The convexity of the corresponding optimization problem is analyzed and an iterative search algorithm is further provided. We study the impact of reliability and short blocklength constraints on the performance of the proposed optimal resource allocation policy through numerical simulations. In addition, we evaluate the FBL performance of the proposed joint design in comparison to the scenario with an IBL assumption.
\end{abstract}

Keywords: Finite blocklength, Resource allocation, SCMA, URLLC

\section{Introduction}

5G new radio is expected to serve ubiquitous applications with excessive data rates and reliability demands for a large number of users. To accomplish this goal, advanced multiple access techniques have been developed while satisfying many requirements such as spectrum efficiency, high system throughput, and massive connectivity. In the last decade, orthogonal frequency division multiple access (OFDMA) [1], which is known as one of the efficient multiple access techniques, has been wellstudied and adopted in the standardization of 3GPP. Recently, non-orthogonal multiple access (NOMA) has been widely investigated, as it allows the network to server more transmissions with limited frequency resource, thus improving the frequency utilization [2-4]. In particular, a new promising NOMA technique, sparse code multiple access (SCMA), has been proposed and investigated $[5,6]$. In general, the SCMA is a codebook-based method where each unique codebook consists of several sparse

\footnotetext{
*Correspondence: hu@ti.rwth-aachen.de

${ }^{2}$ Informationstheorie und Systematischer Entwurf von

Kommunikationssystemen, RWTH Aachen University, Kopernikusstr. 16, 52074 Aachen, Germany

Full list of author information is available at the end of the article
}

codewords that utilize only the corresponding subcarriers. Therefore, a subcarrier in an SCMA network can be shared among different codebooks. Owing to the sparsity of codewords, the multiplexed signals on the same subcarriers can be retrieved with the message passing algorithm (MPA) successfully. Recently, resource allocation has been employed in SCMA networks to improve the system performance. In [7], a swap-matching algorithm is proposed to optimize the codebook assignment and power allocation such that the total sum-rate of the network can be maximized. An optimal resource allocation scheme is introduced for the SCMA network with simultaneous wireless information and power transfer (SWIPT) in [8]. Furthermore, this model is extended to a relay-assisted SCMA network [9] and the resource allocation schemes of SCMA networks for machine-to-machine (M2M) and device-to-device (D2D) communications are discussed in $[10,11]$, respectively. However, all of the above studies on resource allocation in SCMA networks are performed under the ideal assumption of communicating arbitrarily reliable at Shannon's capacity, i.e., codewords are assumed to be of an arbitrary long blocklength.

On the other hand, researchers and designers of nextgeneration wireless networks are increasingly interested 
in having wireless links support delay-sensitive data traffic. In particular, supporting ultra-reliable low latency communications (URLLC) becomes a major concern in the design of $5 G$ networks $[12,13]$. To support URLLC transmissions, most existing works consider OFDMA networks and contribute to the system design. Motivated by the performance advantage (i.e., frequency utilization) of NOMA networks, enabling NOMA systems to support URLLC transmissions has recently emerged and fast developed. In [14, 15], the power domain NOMA-based URLLC for $5 \mathrm{G}$ vehicle-to-everything (V2X) services are introduced and studied. As for the SCMA-based uplink URLLC, the performance of it has been shown is superior to the OFDMA-based one in [16].

However, the above studies and designs for URLLC simply follow Shannon's capacity, i.e., are not taking the impact of the finite blocklength into consideration. It is more accurate to incorporate FBL coding assumptions into the analysis and design of URLLC networks with lowlatency constraints. In such an FBL regime, data transmissions are no longer arbitrarily reliable. Especially when the blocklength is short, the error probability becomes significant even if the rate is selected below the Shannon limit. Taking this into account, an accurate approximation of the achievable coding rate under the finite blocklength assumption for an additive white Gaussian noise (AWGN) channel was studied in $[17,18]$. Following the accurate FBL performance model, multiple user scheduling [19] and power allocation [20] of an OFDMA network has been studied. More recently, the FBL throughput [21] and energy efficiency [22] of power-domain NOMA networks have been discussed. However, the FBL performance and optimal resource allocation for code-domain NOMA networks, e.g., SCMA, have not been addressed so far.

In this paper, we investigate the power allocation and codebook assignment of an SCMA network in URLLC scenario. We first derive the FBL sum-rate of the considered network. Then, a joint optimal design is proposed to maximize the sum-rate, that combines the power allocation with the codebook assignment. The convexity of the corresponding optimization problem is analyzed while an iterative search algorithm is further provided. Via simulations, the impacts of the reliability constraint and the blocklength on the performance of the proposed optimal resource allocation policy are investigated. In particular, we evaluate the FBL performance of the proposed joint design in comparison to the scenario with an infinite blocklength (IBL) assumption. As expected, significant performance differences between the FBL and IBL regimes are observed, which confirms the necessity of adopting the FBL model as the performance indicator in designing a URLLC-supporting SCMA network. More importantly, we find that the optimal resource allocation solutions in the FBL regime and in the IBL regimes are different, i.e., directly applying the optimal resource allocation policy (from existing works) in the IBL regime to a URLLC network results in a considerable performance loss. This again confirms the necessity and contribution of our special design in the FBL regime. Finally, this design is more beneficial for scenarios with a short blocklength or/and a rigorous reliability constraint.

We structure this paper as follows. Section 2 provides a system description and the background of an FBL regime. We propose our optimal resource allocation in the FBL regime in Section 3. The simulation results are provided in Section 4 while the paper is concluded in Section 5.

\section{Preliminaries}

In this section, we describe the system model and provide a brief introduction to the background on an FBL regime.

\subsection{System description}

Consider a downlink multiple access system within a single cell, which consists of only one base station (BS), $K$ user equipments (UEs) and $N$ subcarriers. In the conventional OFDMA system, $N$ must be greater or equal to $K$ to guarantee each UE is at least served by one independent orthogonal subcarrier. In the SCMA network, $N$ can be smaller than $K$ due to the introduction of the non-orthogonality.

In the SCMA system, an SCMA encoder is defined as a map from $\log _{2} M$ binary bits to an $N$-dimensional complex codeword selected from the codebook of size $M$, i.e., $f: \mathbb{B}^{\log _{2} M} \rightarrow \mathcal{X}$, where $\mathcal{X} \subset \mathbb{C}^{N}$ with cardinality $|\mathcal{X}|=M$ and symbols $\mathbb{B}$ and $\mathbb{C}$ represent binary and complex numbers, respectively. Thus, a codebook can be treated as an $N \times M$ matrix. For each codebook, only $J$ subcarriers are active with $J<N$, which makes the codewords sparse. In addition, all the codewords within the same codebook contain zero entries in the same $N-J$ dimensions. Obviously, the maximum number of codebooks $C$ is the number of combinations of $N$ elements choose J, i.e., $C=\left(\begin{array}{l}N \\ J\end{array}\right)$. An example of the codebooks with $M=4, N=4$, and $J=2$ are given in Fig. 1. In this case, there are $C=6$ codebooks in total. Moreover, each codebook consists of four codewords and only occupies two subcarriers. For example, codebook 1 only utilizes the first and the last subcarriers to form the codewords. We use a $C \times N$ matrix $\mathbf{T}=\left\{T_{c, n}\right\}$ to represent the mapping relationship between codebooks and subcarriers. When subcarrier $n$ is employed in codebook $c$, then $T_{c, n}=1$, otherwise $T_{c, n}=0$. And this matrix is predefined as a known parameter. Similarly, the assignment of $C$ codebooks to $K$ users is denoted as a matrix $\mathbf{S}=\left\{S_{k, c}\right\}$ with $S_{k, c}=1$ when codebook $c$ is allocated to user $k$. Note that each codebook can only be assigned to one user. Hence, in order to ensure that every UE can be served by at least one codebook, we need to make the number of UEs less 


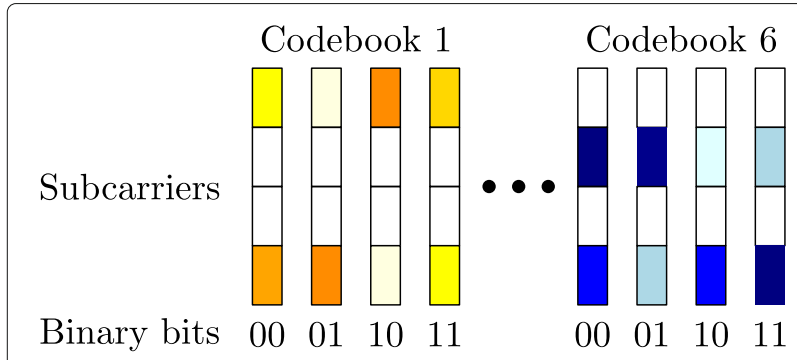

Fig. 1 An example of codebooks

than or equal to the number of codebooks, i.e., $K \leq C$ always holds. An example of mapping relationship among subcarriers, codebooks and users is illustrated in Fig. 2.

In the SCMA network, we let $\mathbf{P}=\left\{P_{k, c}\right\}$ denote the power allocation coefficients with $P_{k, c}$ representing the transmit power from the BS to UE $k$ on codebook $c$. Therefore, the total transmit power of BS is

$$
P^{\text {tot }}=\sum_{k=1}^{K} \sum_{c=1}^{C} S_{k, c} P_{k, c} .
$$

We denote the indices of the utilized $J$ subcarriers for codebook $c$ as set $\mathcal{J}_{c}$, e.g., for codebook 1 given in Fig. 1 , we have $\mathcal{J}_{1}=\{1,4\}$. Thus, the transmit power for UE $k$ with codebook $c$ on subcarrier $n$ is calculated by $P_{k, c, n}=P_{k, c} \alpha_{c, n}$, where $n \in \mathcal{J}_{c}$ and $\alpha_{c, n}$ is a known power allocation fraction for each codebook $c$ on subcarrier $n$. Furthermore, the fractions must meet the requirement $\sum_{n \in \mathcal{J}_{c}} \alpha_{c, n}=1$. Due to the property of the SCMA system, these codebooks can be regarded as orthogonal to each other [8]. Hence, there is no interference between each UE and the signal-to-noise ratio (SNR) of user $k$ on codebook $c$ is given as

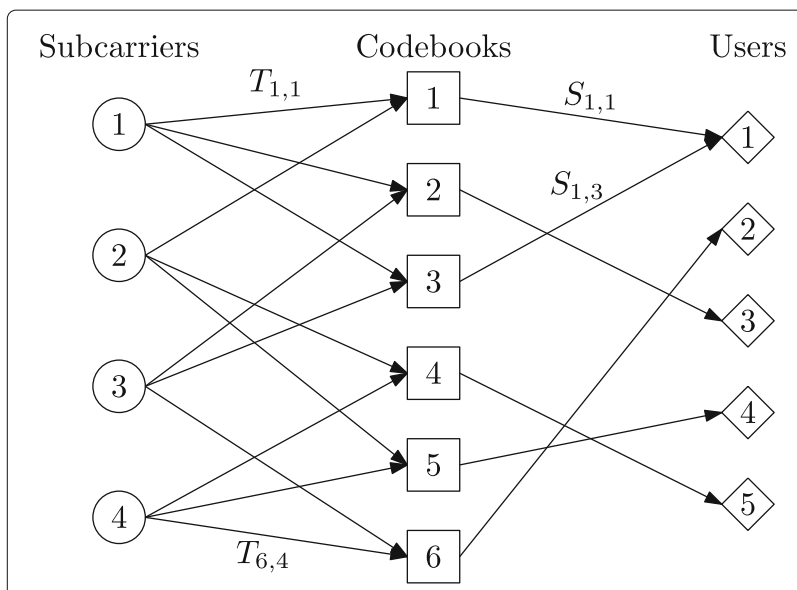

Fig. 2 An example of a mapping scheme in SCMA networks

$$
\gamma_{k, c}=\frac{\sum_{n \in \mathcal{J}_{c}} P_{k, c, n}\left|h_{k, n}\right|^{2}}{\sigma_{k, c}^{2}}=\frac{P_{k, c} \sum_{n \in \mathcal{J}_{c}} \alpha_{c, n}\left|h_{k, n}\right|^{2}}{\sigma_{k, c}^{2}},
$$

where $\sigma_{k, c}^{2}$ is the noise power on codebook $c$ of user $k$ and $h_{k, n}$ is the channel coefficient of user $k$ on subcarrier $n$. In this paper, we consider a quasi-static fading channel; thus, $h_{k, n}$ remains unchanged during a transmission frame.

When compared with the OFDMA, SCMA system can utilize same subcarriers to serve more users. As we know, the OFDMA system employs the orthogonality of the subcarriers to reduce the interference between each user. While the SCMA system utilize the same subcarriers to form more codebooks, then assign these codebooks to each user. These codebooks can be treated as orthogonal as the information conveyed by the codebooks can be decoded with limited interference by MPA successfully [8].

\subsection{Finite blocklength coding}

In $[17,18]$, the authors developed the FBL performance model for a single link transmission by applying a normal approximation. Later on in [23], the third-order term of the normal approximation is further provided for the FBL model. In comparison to the Shannon capacity bound with the IBL assumption, the FBL performance bound is more accurate, especially when the blocklength is short. In particular, for an AWGN channel, the coding rate $\mathcal{R}$ (in bits per channel use) with error probability $0<\epsilon<1$, SNR $\gamma$, and blocklength $v$ is shown to have the following asymptotic expression [23]:

$$
\begin{aligned}
\mathcal{R}(\gamma, \epsilon, v) \approx & \log _{2}(1+\gamma)-\sqrt{\frac{\gamma(\gamma+2)}{(\gamma+1)^{2} v}} \quad Q^{-1}(\epsilon) \log _{2} e \\
& +\frac{\log _{2} v}{v},
\end{aligned}
$$

where $Q(x)=\int_{x}^{\infty} \frac{1}{\sqrt{2 \pi}} e^{-t^{2} / 2} d t$ is the Gaussian $Q$ function.

Note that under a quasi-static fading model, each channel remains constant during a frame. In this work, we will apply the above the FBL coding rate model in our perframe optimal resource allocation. As the approximation has been shown to be accurate for a sufficiently large value of $v[18,23]$, for simplicity we will employ it as the rate expression in our analysis.

Equation (3) facilitates the design in the URLLC networks, as it clearly illustrates the tradeoff between the target error probability $e$ and blocklength $v$ which directly influences the reliability and latency of URLLC transmissions. In particular, to fulfill the ultra-reliability, $\epsilon$ must be set small enough as the lower the error probability is the better the reliability is. On the other hand, the low-latency 
requirement is highly related to $v$ as a smaller blocklength leads to a shorter transmission delay.

\section{Optimal resource allocation}

In this section, we study the optimal resource allocation policy for the considered network. First, the optimization problem is stated. Subsequently, the problem is relaxed and studied. Then, an iterative algorithm is proposed to tackle this problem according to the analysis.

\subsection{Problem statement}

Recall that, we are interested in supporting efficient URLLC transmissions. On the one hand, the served data rate for a UE $k$ should satisfy the basic requirement of the UE, i.e., $R_{k} \geq R_{k}^{\text {req }}$ for a UE $k$. On the other hand, the transmission via each channel is required to be reliable enough, i.e., the error probability of each user's transmission should be lower than a threshold $\epsilon^{\text {th }}$. This condition in terms of channel gain is $\left|h_{k, n}\right|^{2} \geq|h|_{\text {min }}^{2}$, i.e., to support a reliable transmission for user $k$ via a channel $n$, the channel gain $\left|h_{k, n}\right|^{2}$ should be higher than the minimum required channel gain $|h|_{\min }^{2}$. Consequently, when a codebook contains a channel whose channel gain is smaller than the minimum requirement $|h|_{\min }^{2}$, it must be excluded as the transmission is no longer reliable. Hence, the feasible codebook set for a UE $k$ is defined by

$$
\mathcal{C}_{k}=\left\{\left.c|| h_{k, n}\right|^{2} \geq|h|_{\text {min }}^{2}, \forall n \in \mathcal{J}_{c} \text { and } c \in \Omega\right\},
$$

where $\Omega$ is the integer set $\{1,2,3, \ldots, C\}$.

It is possible that all the channels are extremely bad for a UE $k$, i.e., a reliable transmission for the user cannot be guaranteed. Then, we have $\mathcal{C}_{k}=\emptyset$, i.e., no codebooks can serve this user. Thus, this user must be eliminated from the feasible UEs set. We denote by $\mathcal{K}$ the feasible UEs set after the above UE elimination due to the random channel qualities. Then, each feasible UE in the set should be assigned codebooks and allocated power. Note that each UE has a basic data rate requirement and each transmission has a reliability constraint. This generally requires that the SNR of user $k$ on an allocated codebook $c$ cannot be lower than the basic SNR requirement, i.e., $\gamma_{k, c} \geq$ $\gamma_{\min } \geq 0 \mathrm{~dB}$ The $0 \mathrm{~dB}$ lower bound is due to a practical assumption that to support a reliable transmission, the power of the signal at least should not be lower than the noise.

According to (3), the achievable coding rate from the BS to UE $k, k \in \mathcal{K}$, via a codebook $c$ is given by $\mathcal{R}\left(\gamma_{k, c}, \epsilon^{\text {th }}, v\right)$. The throughput (expected amount of bits successfully decoded) of the UE over all assigned codebooks is expressed as

$$
R_{k}=\sum_{c \in \mathcal{C}_{k}} S_{k, c} \mathcal{R}\left(\gamma_{k, c}, \epsilon^{\mathrm{th}}, v\right) \cdot\left(1-\epsilon^{\mathrm{th}}\right)
$$

We consider a general assumption that UEs may have different priorities. We model this by introducing $w_{k}$ as the weighted coefficient for UE $k$. Then, the weighted sum-rate over all UEs is given by $\sum_{k \in \mathcal{K}} w_{k} R_{k}$. It is notable that the URLLC requirements have already been considered in this weighted sum-rate, i.e., the two parameters target error probability $\epsilon$ and blocklength $v$ in (5). The former influences the reliability of the system while the latter affects the latency. Therefore, the maximization problem for optimally assigning codebooks and allocating power of the SCMA network in the FBL regime is formulated as

$$
\begin{array}{ll}
\max _{\mathbf{P}, \mathbf{S}} & \sum_{k \in \mathcal{K}} w_{k} R_{k} \\
\text { s.t. } & \mathrm{C} 1: R_{k} \geq R_{k}^{\mathrm{req}}, \forall k \in \mathcal{K}, \\
& \mathrm{C} 2: P^{\mathrm{tot}} \leq P^{\max }, \\
& \mathrm{C} 3: P_{k, c} \geq \frac{\gamma_{\min } \sigma_{k, c}^{2}}{\sum_{n \in \mathcal{J}_{c}} \alpha_{c, n}\left|h_{k, n}\right|^{2}}, \forall k \in \mathcal{K}, c \in \mathcal{C}_{k}, \\
& \mathrm{C} 4: \sum_{k \in \mathcal{K}} S_{k, c} \leq 1, \forall c \in \mathcal{C}_{k}, \\
& \mathrm{C} 5: S_{k, c} \in\{0,1\}, \forall k \in \mathcal{K}, c \in \mathcal{C}_{k}, \\
& \mathrm{C} 6: \sum_{k \in \mathcal{K}} \sum_{c \in \mathcal{C}_{k}} T_{c, n} S_{k, c} \leq D_{n}, \forall n,
\end{array}
$$

where $D_{n}$ is the maximal reuse time of subcarrier $n$ (by codebooks). $P^{\text {tot }}$ and $P^{\max }$ represent the total and the maximum transmit power of the BS. Moreover, constraint C1 guarantees that each UE can be served with a required rate while $\mathrm{C} 2$ ensures that the maximum transmit power of the BS cannot be exceed. Furthermore, C3 confirms that the SNR of user $k$ on codebook $c$ is greater than the basic SNR requirement. Constrains $\mathrm{C} 4$ and $\mathrm{C} 5$ together restrict that the codebooks are assigned to the UEs exclusively. Finally, C6 guarantees that each subcarrier can be reused with a maximum number $D_{n}$.

\subsection{Resource allocation policy}

Obviously, Problem (6) is a mixed-integer non-convex problem, since the feasible set of $\mathbf{S}$ is not convex. In order to make the problem tractable, we first relax each $S_{k, c}$ from integer to a continuous interval, i.e., $S_{k, c} \in[0,1]$. Furthermore, we employ a new variable $\mathbf{X}=\left\{X_{k, c}\right\}=\left\{S_{k, c} P_{k, c}\right\}$, therefore, the SNR in (2) can be written as

$$
\gamma_{k, c}=\frac{\sum_{n \in \mathcal{J}_{c}} X_{k, c} \alpha_{c, n}\left|h_{k, n}\right|^{2}}{S_{k, c} \sigma_{k, c}^{2}}=\frac{X_{k, c} \beta_{k, c}}{S_{k, c}},
$$


where $\beta_{k, c}=\frac{\sum_{n \in \mathcal{J}_{c}} \alpha_{c, n}\left|h_{k, n}\right|^{2}}{\gamma_{\min } \sigma_{k, c}^{2}}$. Hence, Problem (6) can be transferred to the following problem

$$
\begin{aligned}
& \max _{\mathbf{X}, \mathbf{S}} \sum_{k \in \mathcal{K}} w_{k} R_{k} \\
& \text { s.t. } 1, \mathrm{C} 4, \mathrm{C} 6, \\
& \quad 2^{\prime}: \sum_{k \in \mathcal{K}} \sum_{c \in \mathcal{C}_{k}} X_{k, c} \leq P^{\max }, \\
& \mathrm{C}^{\prime}: X_{k, c} \geq \frac{S_{k, c}}{\beta_{k, c}}, \forall k \in \mathcal{K}, c \in \mathcal{C}_{k}, \\
& \quad \mathrm{C}^{\prime}: 0 \leq S_{k, c} \leq 1, \forall k \in \mathcal{K}, c \in \mathcal{C}_{k},
\end{aligned}
$$

In the following, we propose the optimal resource allocation policy by solving Problem (8). We start with the following proposition illustrating the convexity of Problem (8). Note that the values for blocklength and decoding error probability in the proposition are useful for practical application.

Proposition 1 Problem (8) is a convex optimization problem when $v \geq 100$ and $\epsilon \geq 10^{-20}$.

Proof It holds that the objective function of Problem (8) is a linear combination of the coding rate expression $\mathcal{R}$ under FBL regime. As shown in (3), $\mathcal{R}$ is not a convex function. However, with the given assumption $\gamma \geq \gamma_{\min }=$ $0 \mathrm{~dB}$, it is easy to prove that $\mathcal{R}$ is increasing and concave over $\gamma$ for $v \geq 100$ and $\epsilon \geq 10^{-20}$. The first and second derivatives of $\mathcal{R}$ with respect to $\gamma$ can be calculated as

$$
\frac{\partial \mathcal{R}}{\partial \gamma}=\frac{\log _{2} e}{1+\gamma}\left(1-\frac{\delta}{(1+\gamma) \sqrt{(1+\gamma)^{2}-1}}\right),
$$

$\frac{\partial^{2} \mathcal{R}}{\partial \gamma^{2}}=\frac{\log _{2} e}{(1+\gamma)^{3}}\left(-(1+\gamma)+\frac{\delta}{\left(\gamma^{2}+2 \gamma\right)^{\frac{3}{2}}}+\frac{3 \delta}{\sqrt{\gamma^{2}+2 \gamma}}\right)$,

where $\delta=\sqrt{\frac{1}{v}} Q^{-1}(\epsilon)$, decreasing in $v$ and $\epsilon$. With the given constraints, the maximum value of $\delta$ is $\delta_{\max }=$ 1.085. For the first derivative, considering that $\gamma \geq 1$, the fractional part in the bracket is always smaller than 1, thus, $\frac{\partial \mathcal{R}}{\partial \gamma}>0$ always holds, i.e., the expression of $\mathcal{R}$ is increasing in $\gamma$ with $v \geq 100$ and $\epsilon \geq 10^{-20}$. For the second derivative, when $\epsilon \geq 0.5$, we have $\delta \leq 0$, obviously, $\frac{\partial^{2} \mathcal{R}}{\partial \gamma^{2}}<0$ holds. And when $A>0$, we let $\phi(\gamma)=-(1+\gamma)+\frac{\delta}{2\left(\gamma^{2}+2 \gamma\right)^{\frac{3}{2}}}+\frac{3 \delta}{\sqrt{\gamma^{2}+2 \gamma}}$. Intuitively, $\phi(\gamma)$ is increasing in $\delta$ and thereby decreasing in $v$ and $\epsilon$. Meanwhile, $\phi(\gamma)$ is decreasing in $\gamma$ for $\delta>0$ as well. By considering the condition $\gamma \geq 1$ and substituting $\delta_{\max }$ into $\phi(\gamma)$, we have $\phi(1)=-0.2175<0$. Hence, $\phi(\gamma)<0$ is true for all $\gamma \geq 1$. In other words, $\frac{\partial^{2} \mathcal{R}}{\partial \gamma^{2}}<0$ always holds with the given constraints. Thus, we prove that the coding rate $\mathcal{R}$ is increasing and concave for $\gamma>0 \mathrm{~dB}$ while the blocklength and target error probability are of practical interest, i.e., $v \geq 100, \epsilon \geq 10^{-20}$. Obviously, the convexity of the objective function also holds as it is a linear combination of $\mathcal{R}$. Furthermore, it can be seen that the feasible set of (8) is a convex set. Therefore, Problem (8) is convex.

Proposition 2 When $v \geq 100$ and $\epsilon \geq 10^{-20}$, the equality of the constraint C2' in Problem (8), i.e., $\sum_{k \in \mathcal{K}} \sum_{c \in \mathcal{C}_{k}} X_{k, c}=$ $P^{\max }$, is always required in order to maximize the objective function $\sum_{k \in \mathcal{K}} w_{k} R_{k}$.

Proof We can prove Proposition 2 by contradiction. First of all, we assume that when $v \geq 100$ and $\epsilon \geq 10^{-20}$, there exists an optimal solution $\left(\mathbf{X}^{*}, \mathbf{S}^{*}\right)$ for Problem (8) which satisfies the constraint $\mathrm{C} 2$ ' with a strict inequality, i.e., $\sum_{k \in \mathcal{K}} \sum_{c \in \mathcal{C}_{k}} X_{k, c}^{*}<P^{\max }$. Hence, the remaining power can be obtained and denoted as $P^{\text {rmd }}=P^{\max }-\sum_{k \in \mathcal{K}} \sum_{c \in \mathcal{C}_{k}} X_{k, c}^{*}$ Furthermore, we can partition $P^{\text {rmd }}$ into several pieces, proportionally to $X_{k, c}^{*}$. By denoting these power slices as $X_{k, c}^{\prime}$, we have $\sum_{k \in \mathcal{K}} \sum_{c \in \mathcal{C}_{k}} X_{k, c}^{\prime}=P^{\text {rmd }}$ and $\frac{X_{k, c}^{\prime}}{X_{k, c}^{*}}=\frac{P^{\text {rmd }}}{P^{\text {tot }}}, \forall k \in$ $\mathcal{K}, c \in \mathcal{C}$. In addition, these sliced remainder power $X_{k, c}^{\prime}$ can be added to the optimal ones $X_{k, c}^{*}$ accordingly, thus, form new power symbols denoted as $\hat{X}_{k, c}$, i.e., $\hat{X}_{k, c}=$ $X_{k, c}^{*}+X_{k, c}^{\prime}, \forall k \in \mathcal{K}, c \in \mathcal{C}$. Obviously, the inequality $\hat{X}_{k, c}>$ $X_{k, c}^{*}$ always holds. Thus, we can obtain another solution for Problem (8) by replacing each $X_{k, c}^{*}$ with $\hat{X}_{k, c}$ while keeping the optimal codebook assignment $\mathbf{S}^{\prime}$ unchanged, i.e., $\left(\hat{\mathbf{X}}, S^{*}\right)$. Now, the values of the objective function for these two solutions must be compared. As all the other system parameters stay the same, the value of $\sum_{k \in \mathcal{K}} w_{k} R_{k}$ must increase with $R_{k}$. Furthermore, from Eq. (5), we can see that the value of $R_{k}$ increases with $\mathcal{R}\left(\gamma_{k, c}, \epsilon^{\text {th }}, v\right)$. As proved in Proposition 1, we know that $\mathcal{R}$ is increasing in $\gamma$ when $v \geq 100$ and $\epsilon \geq 10^{-20}$. And as given in Eq. (7), the value of $\gamma_{k, c}$ is increasing in $X_{k, c}$ while keeping $\beta_{k, c}$ and $S_{k, c}$ unchanged. Hence, based on the analysis above, the relationship between the value of the objective function and $X_{k, c}$ can be derived, i.e., $\sum_{k \in \mathcal{K}} w_{k} R_{k}$ is increasing in $X_{k, c}$. As in the proposed new solution $\left(\hat{\mathbf{X}}, S^{*}\right)$ and the assumed optimal solution $\left(\mathbf{X}^{*}, \mathbf{S}^{*}\right)$, the inequality $\hat{X}_{k, c}>$ $X_{k, c}^{*}$ always holds for all $k \in \mathcal{K}$ and $c \in \mathcal{C}$, which leads to the value of the objective function with solution $\left(\hat{\mathbf{X}}, S^{*}\right)$ must be always greater than the one with the optimal solution $\left(\mathbf{X}^{*}, \mathbf{S}^{*}\right)$. Therefore, the assumption that $\left(\mathbf{X}^{*}, \mathbf{S}^{*}\right)$ is 
the optimal solution of Problem (8) is violated and the proposed proposition is proved.

As proven in Proposition 1, Problem (8) is a convex problem under the practical symbol blocklength and error probability constraints, i.e., $v \geq 100$ and $\epsilon \geq 10^{-20}$. We assume that these constraints are always satisfied in the following derivations. Therefore, based on Proposition 2, Problem (8) can be simplified as

$$
\begin{aligned}
& \max _{\mathbf{X}, \mathbf{S}} \sum_{k \in \mathcal{K}} w_{k} R_{k} \\
& \text { s.t. C1, C3', C4, C5', C6 in (8), } \\
& \text { C2" }: \sum_{k \in \mathcal{K}} \sum_{c \in \mathcal{C}_{k}} X_{k, c}=P^{\max } .
\end{aligned}
$$

Since convexity and the Slater's constraint qualification are satisfied by (11), strong duality holds. To increase the convergence speed, the partial Lagrangian method is utilized here, which is constructed as

$$
\begin{aligned}
& \mathcal{L}(\mathbf{X}, \mathbf{S}, \boldsymbol{\lambda}, \mu) \\
& \quad=\sum_{k \in \mathcal{K}}\left[w_{k} R_{k}+\lambda_{k}\left(R_{k}-R^{\mathrm{req}}\right)\right]+\mu\left(P^{\mathrm{max}}-P^{\mathrm{tot}}\right),
\end{aligned}
$$

where $\lambda=\left\{\lambda_{k}, k \in \mathcal{K}\right\}$ is the Lagrange multipliers set for the inequality constraint $\mathrm{C} 1$ and $\mu$ is the multiplier for the equality constraint $\mathrm{C} 2$ ". Therefore, the dual problem is obtained as

$$
\begin{gathered}
\min _{\lambda, \mu} \max _{\mathbf{X}, \mathbf{S}} \mathcal{L}(\mathbf{X}, \mathbf{S}, \boldsymbol{\lambda}, \mu) \\
\text { s.t. } \lambda_{k} \geq 0, \mu \geq 0, \forall k \in \mathcal{K}, c \in \mathcal{C}_{k} \text {, } \\
\text { C3', C4, C5', C6 in (11). }
\end{gathered}
$$

Note that $\mathbf{X}$ and $\mathbf{S}$ in the objective function of Problem (13) can be decoupled and optimized separately as $\max \mathcal{L}=\max \max \mathcal{L}$. This allows us to obtain the corre$\max _{\mathbf{X}, \mathbf{S}} \mathcal{L} \operatorname{Sop}_{\mathbf{X}}$ sponding optimal solutions of $\mathbf{X}$ and $\mathbf{S}$ first, then solve the outer minimization problem with $\lambda$ and $\mu$ subsequently, i.e., Problem (13) can be efficiently solved by applying an iterative algorithm. Consequently, the operations in each iteration loop can be generalized into the following three steps:

\subsubsection{Power allocation}

In the first step, we determine/update the power allocation solution $\mathbf{P}^{*}$. In particular, by solving $\frac{\partial \mathcal{L}}{\partial \mathbf{X}}=0$, we have (14). Based on the given values of $\lambda, \mu$, and $\mathbf{S}$, which are either with initialized values (if this is the first iteration loop) or updated values (from the previous iteration loop), $\mathbf{P}^{*}$ can be obtained/updated by solving (14).

$$
\begin{aligned}
& {\left[\mu-\frac{\beta_{k, c}}{\ln 2}\left(w_{k}+\lambda_{k}\right)\left(\frac{1}{1+P_{k, c} \beta_{k, c}}-\frac{\sqrt{\frac{1}{\nu}} Q^{-1}(\epsilon)}{\left(1+P_{k, c} \beta_{k, c}\right)^{2} \sqrt{\left(1+P_{k, c} \beta_{k, c}\right)^{2}-1}}\right)\right]} \\
& {\left[P_{k, c}-\frac{1}{\beta_{k, c}}\right]=0}
\end{aligned}
$$

The left side of (14) is a product of two terms within two brackets. Obviously, the optimal $P_{k, c}^{*}$ has to at least make the value of either the first term or the second term be 0 . In the following we discuss these cases respectively. To make the first term (in the first bracket) be zero, this requires

$$
\begin{aligned}
& B_{k, c}^{2} y_{k, c}^{6}-2 B_{k, c} y_{k, c}^{5}-\left(B_{k, c}^{2}-1\right) y_{k, c}^{4} \\
&+2 B_{k, c} y_{k, c}^{3}-y_{k, c}^{2}-A^{2}=0,
\end{aligned}
$$

where $y_{k, c}=1+P_{k, c} \beta_{k, c}, A=\sqrt{\frac{1}{v}} Q^{-1}(\epsilon)$, and $B_{k, c}=$ $\frac{\mu \ln 2}{\left(w_{k}+\lambda_{k}\right) \beta_{k, c}}$. Solving (15) with respect to $y_{k, c}$ results in six roots. Denote by $y_{k, c}^{*}$ the optimal solution of $y_{k, c}$. Then, $y_{k, c}^{*}$ should be the one with the largest real value among all the six roots. In particular, as $P_{k, c}$ must be a positive real number, $y_{k, c}^{*}$ should be greater than 1 . Otherwise, there is no solution in this case. As long as we have $y_{k, c}^{*}$, the optimal power is determined, i.e., $\bar{P}_{k, c}^{*}=\left(y_{k, c}^{*}-1\right) / \beta_{k, c}$. On the other hand, Eq. (14) also holds if the second term (on the left side) is zero, i.e., $P_{k, c}-\frac{1}{\beta_{k, c}}=0$. Combined with the power constraint in (8), the optimal power $P_{k, c}^{*}$ under this case is

$$
P_{k, c}^{*}=\left\{\begin{array}{ll}
\bar{P}_{k, c}^{*}, \text { if } \bar{P}_{k, c}^{*} \geq \frac{1}{\beta_{k, c}} \\
\frac{1}{\beta_{k, c}}, \text { if } \bar{P}_{k, c}^{*}<\frac{1}{\beta_{k, c}}
\end{array}, \forall k \in \mathcal{K}, c \in \mathcal{C}_{k} .\right.
$$

\subsubsection{Codebook assignment}

After determining the optimal power $\mathbf{P}^{*}$, the codebook assignment problem can be tackled. By substituting (16) into (12) and eliminating the terms not involving $\mathbf{S}$, the maximization problem with variable $\mathbf{S}$ in (13) can be transferred to a linear program

$$
\begin{aligned}
& \max _{\mathbf{S}} \sum_{k \in \mathcal{K}} \sum_{c \in \mathcal{C}_{k}} Z_{k, c} S_{k, c} \\
& \text { s.t. C4, C5', C6 in (8), }
\end{aligned}
$$

where $Z_{k, c}=\left(w_{k}+\lambda_{k}\right) \mathcal{R}\left(\beta_{k, c} P_{k, c}^{*}, \epsilon^{\text {th }}, v\right)-\mu P_{k, c}^{*}, \forall k, c$. It should be noted that $C 6$ is guaranteed by the predefined mapping matrix $\mathbf{T}$ between codebooks and subcarriers when the constraints $\mathrm{C} 4$ and $\mathrm{C}^{\prime}$ are satisfied. And the optimal solution of problem (17) is given by

$$
S_{k, c}^{*}=\left\{\begin{array}{l}
1, \text { if } k=\arg \max _{k \in \mathcal{K}}\left\{Z_{k, c}\right\}, \\
0, \text { otherwise }
\end{array},\right.
$$

$\forall k \in \mathcal{K}, c \in \mathcal{C}_{k}$. Thus, the optimal codebook assignment matrix $\mathbf{S}^{*}$ is obtained. 


\subsubsection{Subgradient method}

As $\mathbf{P}^{*}$ and $\mathbf{S}^{*}$ have been obtained, in the last step, we have to solve the outer minimization problem in (13). It has been proven $[8,9]$ that applying the subgradient method can efficiently achieve the dual solutions. The subgradient method is an iterative method for tackling the convex minimization problems and can ensure the convergence when adopting to non-differentiable objective functions. In details, the update processes for $\lambda_{k}, k \in \mathcal{K}$ and $\mu$ are

$$
\begin{aligned}
& \lambda_{k}(t+1)=\left[\lambda_{k}(t)-\varphi_{k}(t)\left(R_{k}(t)-R^{\mathrm{req}}\right)\right]^{+}, \\
& \mu(t+1)=\left[\mu(t)-\psi(t)\left(P^{\max }-P^{\mathrm{tot}}(t)\right)\right]^{+},
\end{aligned}
$$

where $\varphi_{k}(t)$ and $\psi(t)$ are small positive step sizes of $\lambda_{k}$ and $\mu$ in the $t^{\text {th }}$ iteration, respectively. It has been shown that the convergence can be guaranteed with properly selected step sizes $[8,9]$.

Combining the above three steps, we provide in Algorithm 1 the overall resource allocation policy solving problem (6).

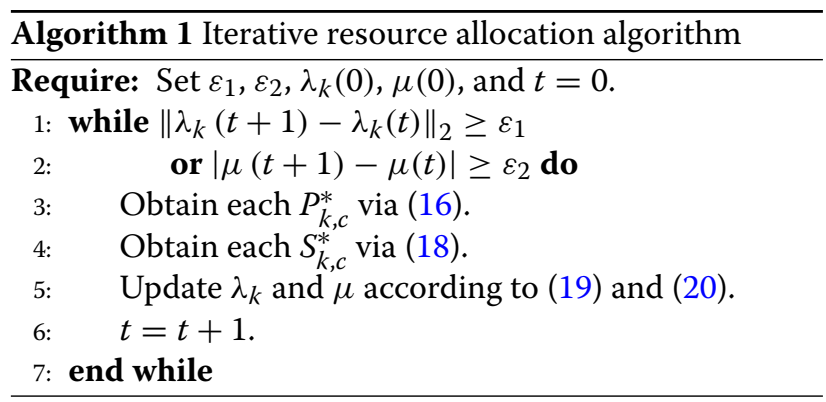

\section{Simulation results and discussion}

We resort to Monte Carlo simulations to evaluate the performance of the proposed algorithm and to study how the blocklength, the target error probability and the SNR effect the FBL throughput. In the simulation, we compare the throughput performance of the proposed algorithm in the FBL regime with existing works which consider maximizing the Shannon capacity in the IBL regime. In particular, two groups of reference performances are provided. One is called "Shannon" results, i.e., the Shannon capacity in the IBL regime. The other one is called "Mixed" scheme results, which is the FBL throughput obtained by directly applying the optimal resource allocation solutions maximizing the Shannon capacity (in the IBL regime) to the FBL regime.

In all simulations, an SCMA downlink network with 1 BS and 5 UEs is considered. The Rayleigh multipath fading is employed here. We let the average channel gain for each UE is $|h|^{2}=1$ and set the minimum required channel gain $|h|_{\min }^{2}=0.01$. Thus, less than $1 \%$ UE cannot be served. Meanwhile, we set the path loss for each link to $80 \mathrm{~dB}$. The noise power is $\delta=-80 \mathrm{dBm}$. And we let the average transmit power for each user be equal to $5 \mathrm{dBm}$. In terms of SCMA, we set $N=4$ and $J=2$. Thus, the total available codebook is $C=\left(\begin{array}{c}N \\ J\end{array}\right)=6$. In addition, the power allocation fraction is $\alpha_{c, n}=0.5$ when the subcarrier $n$ is used by the codebook $c, \forall c \in\{1,2, \ldots, C\}, n \in$ $\{1,2, \ldots, N\}$. Finally, we have a homogeneous assumption on UEs' requirements, i.e., $R_{k}^{\text {req }}=1$ bits/ch.use and $w_{k}=$ 1.

To start with, the relationship between target error probability and throughput is illustrated in Fig. 3. The blocklength is set to 100 symbols, i.e., corresponding to a latency critical scenario. Then, Fig. 3 actually demonstrates under a latency critical scenario the cost of guaranteeing a certain level of reliability with respects to the throughput. It can be observed that the Shannon capacity is constant in the error probability while the FBL throughputs of the mixed and the proposed schemes are increasing in the error probability. More importantly, the achievable performances under the FBL and IBL regimes (FBL throughputs vs. Shannon capacity) are significantly different. Moreover, there is also a considerable gap between the FBL throughput of the proposed algorithm and the one with the mixed scheme. In other words, directly applying the optimal resource allocation policy in the IBL regime to a URLLC network results in a considerable throughput loss. Finally, the above two performance gaps are decreasing in the error probability.

Next, we analyze in Fig. 4 the impact of the blocklength on the throughput performance. The error probability is set to $10^{-5}$, which ensures $99.999 \%$ system transmission correctness. In addition, note that in a URLLC network design, the (maximal allowed) blocklength is affine in the

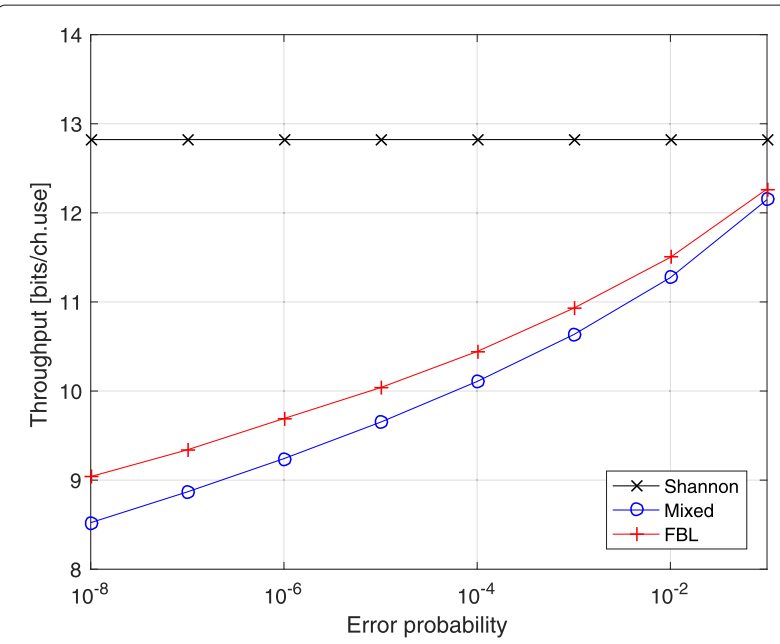

Fig. 3 Throughput vs. target error probability with blocklength $v=100$ 


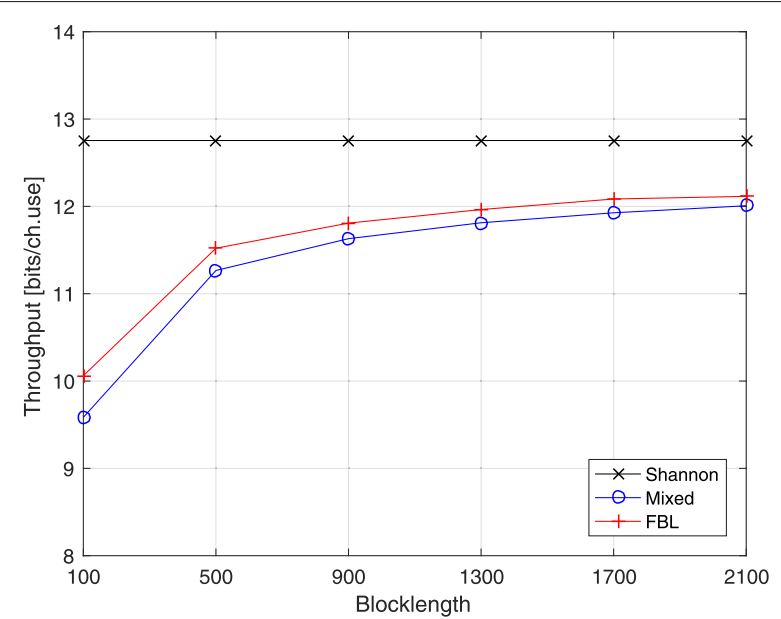

Fig. 4 Throughput vs. blocklength with target error probability $\epsilon=10^{-5}$

latency requirement. Hence, the results in Fig. 4 indicate the costs (with respects to throughput) of guaranteeing reliably of $99.999 \%$ under different latency constraints. Again, significant performance differences among the Shannon capacity, the FBL throughputs of the proposed policy, and the mixed scheme in the IBL regime are observed. These differences are reduced as the blocklength increases. This holds as the longer the blocklength is, the closer it is to the infinite blocklength. Thus, the performance gain achieved by considering the blocklength into the system design becomes less. Finally, we have done the same simulations with different setups of SNRs. The plots in these cases are quite similar to the plots in Figs. 3 and 4 and not provided in the paper, while it should be mentioned that the performance gaps between FBL and IBL regimes become relatively small in a high SNR region.

In addition to the throughput, spectral efficiency and energy efficiency are also interesting performance indicators in the SCMA network design. In the system we considered in this work, all subcarriers have the same frequency occupancy. Hence, the plots for spectral efficiency against error probability or blocklength are just a scaling on the $y$-axis of Figs. 3 and 4. On the other hand, for the energy efficiency, based on Proposition 2, we can derive that the energy consumption of all these schemes are the same, i.e., the maximum. Therefore, similar to the case for spectral efficiency, the energy efficiency can be treated as a scaling factor as well. In summary, both the energy and the spectral efficiency performance of these schemes are just scaling of their throughput plots. Hence, the proposed scheme always outperforms the mix-scheme with respects to throughput, spectral efficiency and energy efficiency.

\section{Conclusions}

In this work, we study an SCMA network supporting URLLC transmissions in the FBL regime. An optimal resource allocation policy is proposed to maximize the throughput. Via simulations, we evaluate the (achievable) FBL throughput performance of the considered network with the proposed resource allocation policy. In particular, we compare this (achievable) FBL throughput with the Shannon capacity in the IBL regime and with the FBL throughput obtained by applying the optimal solution of maximizing the Shannon capacity to the FBL model. Significant performance differences between these throughputs/capacity are observed, which confirm the necessity and contribution of this study: First, the system designs following the Shannon capacity are not accurate for the design of SCMA networks supporting URLLC transmissions. Moreover, a special design as provided in this work is necessary as the optimal solutions in the FBL regime and in the IBL regimes are different, i.e., directly applying the optimal resource allocation policy (from existing works) in the IBL regime to a URLLC network results in a considerable performance loss. Finally, this special design in the FBL regime is more beneficial for scenarios with a short blocklength or/and a rigorous reliability constraint.

In our future work, we will consider to employ the game theory-based resource allocation schemes, e.g., the swapmatching algorithm [7], to SCMA networks and provide designs to enable the URLLC transmissions.

\section{Abbreviations}

AWGN: Additive white Gaussian noise; BS: Base station; D2D: Device-to-device; FBL: Finite blocklength; IBL: Infinite blocklength; M2M: Machine-to-machine; MPA: Message passing algorithm; NOMA: Non-orthogonal multiple access; OFDMA: Orthogonal frequency division multiple access; SCMA: Sparse code multiple access; SNR: Signal-to-noise ratio; SWIPT: Simultaneous wireless information and power transfer; UE: User equipment; URLLC: Ultra-reliable low latency communications; V2X: Vehicle-to-everything

\section{Funding}

This work is supported by Deutsche Forschungsgemeinschaft (DFG) research grant SCHM 2643/13-1 and RWTH startup grant StUpPD 262-17.

\section{Availability of data and materials}

The paper is self-contained. Simulation description and parameters are provided in details in Section 4.

\section{Authors' contributions}

All authors have contributed to this manuscript and approved the submitted manuscript.

\section{Competing interests}

The authors declare that they have no competing interests.

\section{Publisher's Note}

Springer Nature remains neutral with regard to jurisdictional claims in published maps and institutional affiliations.

\section{Author details}

${ }^{1}$ ISEK Research Group, RWTH Aachen University, Kopernikusstr. 16, 52074 Aachen, Germany. ${ }^{2}$ Informationstheorie und Systematischer Entwurf von Kommunikationssystemen, RWTH Aachen University, Kopernikusstr. 16, 52074 Aachen, Germany.

Received: 6 September 2018 Accepted: 16 November 2018

Published online: 06 December 2018 


\section{References}

1. Y. Sun, C. Li, Y. Hung, L. Yang, Joint Fronthaul Rate Allocation and Power Control in OFDMA-based C-RANs: Multi-Objective Approach. Adhoc Sens Wirel. Netw. 37(1-4), 197-229 (2017). 33p

2. Y. Liu, Z. Qin, M. Elkashlan, Z. Ding, A. Nallanathan, L. Hanzo, Nonorthogonal multiple access for $5 \mathrm{~g}$ and beyond. Proc. IEEE. 105(12), 2347-2381 (2017). https://doi.org/10.1109/JPROC.2017.2768666

3. Z. Chang, L. Lei, H. Zhang, T. Ristaniemi, S. Chatzinotas, B. Ottersten, Z. Han, Energy-efficient and secure resource allocation for multiple-antenna NOMA with wireless power transfer. IEEE Trans. Green Commun. Networking, 1-1 (2018). https://doi.org/10.1109/TGCN.2018.2851603

4. T. Qi, W. Feng, Y. Chen, Y. Wang, When NOMA meets sparse signal processing: Asymptotic performance analysis and optimal sequence design. IEEE Access. 5, 18516-18525 (2017). https://doi.org/10.1109/ ACCESS.2017.2727518

5. H. Nikopour, H. Baligh, in 2013 IEEE 24th Annual International Symposium on Personal, Indoor, and Mobile Radio Communications (PIMRC), London. Sparse code multiple access (IEEE, 2013), pp. 332-336. https://doi.org/10. 1109/PIMRC.2013.6666156

6. M. Taherzadeh, H. Nikopour, A. Bayesteh, H. Baligh, in 2014 IEEE 80th Vehicular Technology Conference (VTC2014-Fall), Vancouver, BC. SCMA Codebook Design (IEEE, 2014), pp. 1-5. https://doi.org/10.1109/NTCFall. 2014.6966170

7. B. Di, L. Song, Y. Li, in 2016 IEEE International Conference on Communications (ICC), Kuala Lumpur. Radio resource allocation for uplink sparse code multiple access (SCMA) networks using matching game (IEEE, 2016), pp. 1-6. https://doi.org/10.1109/ICC.2016.7511409

8. D. Zhai, M. Sheng, X. Wang, Y. Li, J. Song, J. Li, Rate and energy maximization in scma networks with wireless information and power transfer. IEEE Commun. Lett. 20(2), 360-363 (2016). https://doi.org/10 1109/LCOMM.2015.2504439

9. L. Luo, L. Li, X. Su, in 2017 International Conference on Computing Networking and Communications (ICNC), Santa Clara, CA. Optimization of resource allocation in relay assisted multi-user SCMA uplink network (IEEE, 2017), pp. 282-286. https://doi.org/10.1109/ICCNC.2017.7876140

10. J. Shang, L. Gui, H. Zhou, M. Dong, J. Chen, in 20168 th International Conference on Wireless Communications \& Signal Processing (WCSP), Yangzhou. A group-based M2M multiple access scheme in massive MIMO MU-SCMA cellular networks (IEEE, 2016), pp. 1-6. https://doi.org/10.1109/ WCSP.2016.7752657

11. D. Zhai, M. Sheng, X. Wang, Z. Sun, C. Xu, J. Li, Energy-saving resource management for D2D and cellular coexisting networks enhanced by hybrid multiple access technologies. IEEE Trans. Wirel. Commun. 16(4), 2678-2692 (2017). https://doi.org/10.1109/TWC.2017.2671863

12. C. She, C. Yang, T. Q. S. Quek, Radio resource management for ultra-reliable and low-latency communications. IEEE Commun. Mag 55(6), 72-78 (2017). https://doi.org/10.1109/MCOM.2017.1601092

13. Y. Hu, M. C. Gursoy, A. Schmeink, Relaying-enabled ultra-reliable low-latency communications in 5G. IEEE Netw. 32(2), 62-68 (2018). https://doi.org/10.1109/MNET.2018.1700252

14. B. Di, L. Song, Y. Li, G. Y. Li, in GLOBECOM 2017 - 2017 IEEE Global Communications Conference, Singapore. NOMA-based low-latency and high-reliable broadcast communications for $5 G$ V2X services (IEEE, 2017), pp. 1-6. https://doi.org/10.1109/GLOCOM.2017.8254830

15. B. Di, L. Song, Y. Li, Z. Han, V2X meets NOMA: Non-orthogonal multiple access for 5G-enabled vehicular networks. IEEE Wirel. Commun. 24(6), 14-21 (2017). https://doi.org/10.1109/MWC.2017.1600414

16. C. Wang, Y. Chen, Y. Wu, L. Zhang, in 2017 IEEE 85th Vehicular Technology Conference (VTC Spring), Sydney, NSW. Performance evaluation of grant-free transmission for uplink URLLC services (IEEE, 2017), pp. 1-6. https://doi.org/10.1109/NTCSpring.2017.8108593

17. Y. Polyanskiy, H. V. Poor, S. Verdu, in 2009 IEEE International Symposium on Information Theory, Seoul. Dispersion of Gaussian channels (IEEE, 2009), pp. 2204-2208. https://doi.org/10.1109/ISIT.2009.5205834

18. Y. Polyanskiy, H. V. Poor, S. Verdu, Channel coding rate in the finite blocklength regime. IEEE Trans. Inf. Theory. 56(5), 2307-2359 (2010). https://doi.org/10.1109/TIT.2010.2043769

19. S. Xu, T. Chang, S. Lin, C. Shen, G. Zhu, Energy-efficient packet scheduling with finite blocklength codes: Convexity analysis and efficient algorithms. IEEE Trans. Wirel. Commun. 15(8), 5527-5540 (2016). https://doi.org/10. 1109/TWC.2016.2561273
20. Y. Hu, M. Ozmen, M. C. Gursoy, A. Schmeink, in 2018 IEEE Wireless Communications and Networking Conference (WCNC), Barcelona. Optimal power allocation for QoS-constrained downlink networks with finite blocklength codes (IEEE, 2018), pp. 1-6. https://doi.org/10.1109/WCNC. 2018.8377030

21. Y. Hu, M. C. Gursoy, A. Schmeink, in 2017 IEEE 28th Annual International Symposium on Personal, Indoor, and Mobile Radio Communications (PIMRC), Montreal, QC. Efficient transmission schemes for low-latency networks: NOMA vs. relaying (IEEE, 2017), pp. 1-6. https://doi.org/10.1109/PIMRC. 2017.8292490

22. Y. Xu, C. Shen, T. Chang, S. Lin, Y. Zhao, G. Zhu, in 2017 IEEE Globecom Workshops (GC Wkshps), Singapore. Energy-efficient non-orthogonal transmission under reliability and finite blocklength constraints (IEEE, 2017), pp. 1-6. https://doi.org/10.1109/GLOCOMW.2017.8269136

23. V. Y. F. Tan, M. Tomamichel, The third-order term in the normal approximation for the AWGN channel. IEEE Trans. Inf. Theory. 61(5), 2430-2438 (2015). https://doi.org/10.1109/TIT.2015.2411256

\section{Submit your manuscript to a SpringerOpen ${ }^{\circ}$ journal and benefit from:}

- Convenient online submission

- Rigorous peer review

- Open access: articles freely available online

- High visibility within the field

- Retaining the copyright to your article

Submit your next manuscript at $\$$ springeropen.com 